\title{
Relations and non-commutative linear logic
}

\author{
Carolyn Brown and Doug Gurr \\ University of Århus*
}

November 1991

\section{Introduction}

Linear logic [Gir87, GL87] differs from intuitionistic logic primarily in the absence of the structural rules of weakening and contraction. Weakening allows us to prove a proposition in the context of irrelevant or unused premises, while contraption allows us to use a premise an arbitrary number of times. Linear logic has been galled a "resource-conscious logic", since the premises of a sequent must appear exactly as many times as they are used.

In non-commutative linear logic, the structural rule of exchange, which allows one to permute the order of premises, is also discarded. Girard [Gir89] has stated that,

" ... [the Lambeck calculus'] rejection of exchange seem[s] to indicate that, eventually, linear logic should be non-commutative"

and continues in the same article to refer to

" ...non-commutative linear logic, which should play a prominent role in the future, but which is still very experimental."

*This work has been supported by ESPRIT CEDISYS and CLICS and by the Danish DART. 
In this paper, we address this "experimental nature" by presenting a noncommutative intuitionistic linear logic with several attractive properties. Our logic discards even the limited commutativity of Yetter's logic [Yet90] in which eyelid permutations of formulae are permitted. It arises in a natural way from the system of intuitionistic linear logic presented in [GL87], and we prove a cut elimination theorem. In linear logic, the rules of weakening and contraction are recovered in a restricted sense by the introduction of the exponential modality (!). This recaptures the expressive power of intuitionistic logic. In our logic the modality! recovers the non-commutative analogues of these structural rules. However, the most appealing property of our logic is that it is both sound and complete with respect to interpretation in a natural class of models which we call relational quantales.

Quantales are a generalisation of locales, introduced by Mulvey [Mul86] with the aim of providing a constructive formulation of the foundations of quantum mechanics. Interest in quantales has recently been stimulated by the fact that intuitionistic linear logic can be interpreted in any commutative quantale, and that commutative quantales constitute a sound and complete class of models [Yet90].

We study relational quantales, in which the elements are relations on a set $A$ ordered by inclusion, and the monoid operation is relational composition. Such structures have been proposed by Hoare and He Jefing [HH87] as models for the semantics of non-deterministic while programs and for program specification. Our central result is that relational quantales provide a sound and complete class of models for our non-commutative intuitionistic linear logic. The result rests on a representation theorem [BG91] for quantales which states that every quantale is isomorphic to a relational quantale.

The value of this result is that it provides natural models in which the noncommutative $\otimes$ is interpreted by the familiar notion of relational composition and the exponential ! $A$ has a natural interpretation as the largest reflexive and transitive relation contained in $A$. In addition, it suggests that noncommutative linear logic may be a suitable language to describe the structures proposed by Hoare and He Jefing. 


\section{Non-commutative Intuitionistic Linear Logic}

In [GL87], Girard and Lafont present the following system of intuitionistic linear logic.

$$
\begin{aligned}
& \overline{A \vdash A} \text { (Id) } \frac{\Gamma \vdash A \Delta, A \vdash B}{\Gamma, \Delta \vdash B} \text { (Cut) } \frac{\Gamma, A, B, \Delta \vdash C}{\Gamma, B, A, \Delta \vdash C} \text { (Ex) } \\
& \overline{\Gamma, \mathbf{0} \vdash A}(\mathbf{0} \vdash) \quad \overline{\Gamma \vdash \mathbf{1}}(\vdash \mathbf{1}) \quad \frac{\Gamma \vdash A}{\Gamma, \mathrm{I} \vdash A}(\mathrm{I} \vdash) \quad \overline{\vdash \mathrm{I}}(\vdash \mathrm{I}) \\
& \frac{\Gamma \vdash A \Delta \vdash B}{\Gamma, \Delta \vdash A \otimes B}(\vdash \otimes) \quad \frac{\Gamma, A, B \vdash C}{\Gamma, A \otimes B \vdash C}(\otimes \vdash) \\
& \frac{\Gamma \vdash A}{\Gamma \vdash A \otimes B}(\vdash \otimes 1) \quad \frac{\Gamma, A, B \vdash C}{\Gamma, A \otimes B \vdash C}(\otimes \vdash \mathrm{r}) \\
& \frac{\Gamma, A \vdash C \Gamma, B \vdash C}{\Gamma, A \oplus B \vdash C}(\otimes \vdash) \quad \frac{\Gamma, A \Gamma \vdash B}{\Gamma \vdash A \wedge B}(\vdash \wedge) \\
& \frac{\Gamma, A \vdash C}{\Gamma, A \wedge B \vdash C}(1 \wedge \vdash) \quad \frac{\Gamma, B \vdash C}{\Gamma, A \wedge B \vdash C}(\mathrm{r} \wedge \vdash) \\
& \frac{\Gamma \vdash A \Delta, B \vdash C}{\Gamma, \Delta, A \multimap B \vdash C}(\multimap \vdash) \quad \frac{\Gamma, A \vdash B}{\Gamma \vdash A \multimap B}(\vdash \multimap)
\end{aligned}
$$

Non-commutative intuitionistic linear logic (NILL) is obtained by discarding the rule of exchange and making the appropriate modifications to the remaining rules. The rules of NILL are as follows.

$$
\overline{A \vdash A} \text { (Id) } \frac{\Gamma \vdash A \Delta, A, \Delta^{\prime} \vdash B}{\Delta, \Gamma, \Delta^{\prime} \vdash B} \text { (Cut) }
$$




$$
\begin{aligned}
& \overline{\Gamma, \mathbf{0}, \Delta \vdash A}(\mathbf{0} \vdash) \quad \overline{\Gamma \vdash \mathbf{1}}(\vdash \mathbf{1}) \quad \frac{\Gamma, \Delta \vdash A}{\Gamma, \mathrm{I}, \Delta \vdash A}(\mathrm{I} \vdash) \quad \overline{\vdash \mathrm{I}}(\vdash \mathrm{I}) \\
& \frac{\Gamma \vdash A \Delta \vdash B}{\Gamma, \Delta \vdash A \otimes B}(\vdash \otimes) \quad \frac{\Gamma, A, B, \Delta \vdash C}{\Gamma, A \otimes B, \Delta \vdash C}(\otimes \vdash) \\
& \frac{\Gamma \vdash A}{\Gamma \vdash A \otimes B}(\vdash \otimes 1) \quad \frac{\Gamma \vdash B}{\Gamma, A \otimes B}(\vdash \oplus \mathrm{r}) \\
& \frac{\Gamma, A, \Delta \vdash C \Gamma, B, \Delta \vdash C}{\Gamma, A \oplus B, \Delta \vdash C}(\otimes \vdash) \quad \frac{\Gamma \vdash A \Gamma \vdash B}{\Gamma \vdash A \wedge B}(\vdash \wedge) \\
& \frac{\Gamma, A, \Delta \vdash C}{\Gamma, A \wedge B, \Delta \vdash C}(1 \wedge \vdash) \quad \frac{\Gamma, B, \Delta \vdash C}{\Gamma, A \wedge B, \Delta \vdash C}(\mathrm{r} \wedge \vdash) \\
& \frac{\Gamma \vdash A \Delta, B, \Delta^{\prime} \vdash C}{\Delta, A \multimap \multimap_{\mathrm{r}} B, \Gamma, \Delta^{\prime} \vdash C}\left(\multimap_{\mathrm{r}} \vdash\right) \quad \frac{\Gamma, A \vdash B}{\Gamma \vdash A \multimap_{\mathrm{r}} B}\left(\vdash \multimap_{\mathrm{r}}\right) \\
& \frac{\Gamma \vdash A \Delta, B, \Delta^{\prime} \vdash C}{\Delta, \Gamma, A \multimap \multimap_{1} B, \Delta^{\prime} \vdash C}\left(\multimap_{1} \vdash\right) \quad \frac{A, \Gamma \vdash B}{\Gamma \vdash A \multimap 1}(\vdash \multimap 1)
\end{aligned}
$$

Non-commutative linear logic differs from linear logic primarily in the absence of the exchange rule:

$$
\frac{\Gamma, A, B, \Delta \vdash C}{\Gamma, B, A, \Delta \vdash C}(\mathrm{Ex})
$$

Thus in NILL the multiplicative connective $\otimes$ and the comma of the left hand side of a sequent are non-commutative. The additive connectives $\wedge$ and $\oplus$ remain commutative. There are two main consequences of discarding the exchange rule. Firstly, we replace linear implication by two implications, 
$\multimap_{1}$ and $\multimap_{\mathrm{r}}$, whose rules of introduction and elimination differ subtly. The following derived theorems illustrate the difference between these two implications:

$$
\overline{A, A \multimap \multimap_{1} B \vdash B} \quad \overline{A \multimap \multimap_{\mathrm{r}} B, A \vdash B}
$$

Secondly, the position of a formula in the left hand side of a sequent becomes significant, and we must modify certain rules accordingly. Thus the rules $(\mathbf{0} \vdash),(\mathrm{I} \vdash),(\oplus \vdash),(\otimes \vdash)(\mathrm{r} \wedge \vdash),(\mathrm{l} \wedge \vdash)$ and $(\mathrm{Cut})$ each have an additional $\Delta$ in their premises and conclusions There are no other modifications.

Remark 2.1 In fact, our cut rule is derivable from the apparently weaker rule:

$$
\frac{\Gamma \vdash A A \vdash B}{\Gamma \vdash B}\left(\mathrm{Cut}^{\prime}\right)
$$

Proof: Suppose that $\Gamma \vdash A$ and $\Delta, A, \Delta^{\prime} \vdash B$ where $\Delta=\Delta_{1}, \Delta_{2}, \ldots, \Delta_{n}$ and $\Delta^{\prime}=\Delta_{1}^{\prime}, \Delta_{2}^{\prime}, \ldots, \Delta_{m}^{\prime}$. Then by repeated applications of the rule $(\vdash \otimes)$ we obtain

$$
\begin{aligned}
& \begin{array}{c}
\frac{\overline{\Delta_{n} \vdash \Delta_{n}}}{\Delta_{n-1} \vdash \Delta_{n-1}} \quad \frac{\Delta_{n}, \Gamma \vdash \Delta_{n} \otimes A}{\Delta_{n-1}, \Delta_{n}, \Gamma \vdash \Delta_{n-1} \otimes \Delta_{n} \otimes A} \\
\frac{\Delta_{\Delta_{n}}}{\Delta_{n}}
\end{array} \\
& \begin{array}{cl}
\Delta, \Gamma \vdash \Delta_{1} \otimes \Delta_{2} \otimes \cdots \otimes \Delta_{n} \otimes A & \Delta_{1}^{\prime} \vdash \Delta_{1}^{\prime} \\
\hline \Delta, \Gamma, \Delta_{1}^{\prime} \vdash \Delta_{1} \otimes \Delta_{2} \otimes \cdots \otimes \Delta_{n} & \otimes A \otimes \Delta_{1}^{\prime}
\end{array} \\
& \vdots \\
& \Delta, \Gamma, \Delta^{\prime} \vdash \Delta_{1} \otimes \Delta_{2} \otimes \cdots \otimes \Delta_{n} \otimes A \quad \otimes \Delta_{1}^{\prime} \otimes \cdots \otimes \Delta_{m}^{\prime}
\end{aligned}
$$

and by repeated applications of the rule $(\otimes \vdash)$ we obtain

$$
\frac{\frac{\Delta_{1}, \Delta_{2}, \ldots, \Delta_{n}, A, \Delta^{\prime} \vdash B}{\frac{\Delta_{1} \otimes \Delta_{2} \ldots \Delta_{n}, A, \Delta^{\prime} \vdash B}{\vdots}}}{\frac{\vdots}{\Delta_{1} \otimes \Delta_{2} \otimes \cdots \otimes \Delta_{n} \otimes A \otimes \Delta_{1}^{\prime} \otimes \cdots \Delta_{m}^{\prime} \vdash B}}
$$

and finally we obtain the sequent $\Delta, \Gamma, \Delta^{\prime} \vdash B$ by applying the rule Cut' to the formula $\Delta_{1} \otimes \Delta_{2} \otimes \cdots \Delta_{n} \otimes A \otimes \Delta_{1}^{\prime} \otimes \cdots \otimes \Delta_{m}^{\prime}$. 
In particular, we could have taken the cut rule from the system of Girard and Lafont. However, it is technically convenient to take our cut rule as primitive.

\section{Cut Elimination}

We prove the central logical result, that the cut rule is redundant. We assume standard definitions of the height $h(\pi)$ of a proof $\pi$ and the degree of a formula [GLT89]. The degree of a cut rule is the degree of the cut formula and the degree of a proof is the maximum degree of its cut rules.

Lemma 3.1 Let $C$ be a formula of degree $d$ and $\pi, \pi^{\prime}$ proofs of degree $<d$ of $\Gamma \vdash C$ and $\Delta_{0}, C, \Delta_{1} \vdash B$ respectively. Then there is a proof of $\Delta_{0}, \Gamma, \Delta_{1} \vdash B$ of degree $<d$.

Proof: We proceed by induction on $h(\pi)+h\left(\pi^{\prime}\right)$ and consider the possible last rules $r$ and $r^{\prime}$ of $\pi$ and $\pi^{\prime}$ resnectivelv.

- If $r$ is (id) we use the proof $\pi^{\prime}$.

- If $r^{\prime}$ is (id) we use the proof $\pi$.

- If $r$ is

$$
\frac{\Gamma_{1} \vdash D \Gamma_{0}, D, \Gamma_{2} \vdash C}{\Gamma_{0}, \Gamma_{1}, \Gamma_{2} \vdash C} \text { (Cut) }
$$

we apply the inductive hypothesis to the proof of $\Gamma_{0}, D, \Gamma_{2} \vdash C$ and $\pi^{\prime}$ to obtain a proof of $\Delta_{0}, \Gamma_{0}, D, \Gamma_{2}, \Delta_{1} \vdash B$ of degree $<d$. We then cut on the formula $D$ which is of degree $<d$ since it is cut on in the proof $\pi$ which has degree $<d$, by the hypotheses of the lemma.

- If $r^{\prime}$ is

$$
\frac{\Gamma_{1} \vdash D \Gamma_{0}, D, \Gamma_{2}, C, \Gamma_{3} \vdash B}{\Gamma_{0}, \Gamma_{1}, \Gamma_{2}, C, \Gamma_{3} \vdash B}(\mathrm{Cut})
$$

we apply the inductive hypothesis to $\pi$ and the proof of $\Gamma_{0}, D, \Gamma_{2}, C, \Gamma_{3} \vdash$ $B$ to obtain a proof of $\Gamma_{0}, D, \Gamma_{2}, \Gamma, \Gamma_{3} \vdash B$ of degree $<d$. We then cut on the formula $D$ which is of degree $<d$ since it is cut on in the proof $\pi^{\prime}$ which has degree $<d$, by the hypotheses of the lemma. 
- If $r$ is ( $\vdash \vdash)$ for some connective $\circ$ we apply the inductive hypothesis where appropriate to $\pi^{\prime}$ and to the proofs $\pi_{i}$ of the premises of $r$, and apply $r$ to the resulting proofs.

- If $r^{\prime}$ is $(\vdash \circ)$ for some connective $\circ$ we apply the inductive hypothesis where appropriate to $\pi$ and to the proofs $\pi_{j}^{\prime}$ of the premises of $r^{\prime}$, and apply $r^{\prime}$ to the resulting proofs.

The final (and key) case is where $r$ is $(\vdash \circ)$ and $r^{\prime}$ is $(\circ \vdash)$ for some connective $\circ$. We show how to transform each of the possible cases.

$$
\begin{aligned}
& \frac{\mp \quad \frac{\Delta_{0}, \Delta_{1} \vdash C}{\Delta_{0}, \mathrm{I}, \Delta_{1} \vdash C}}{\Delta_{0}, \Delta_{1} \vdash C} \leadsto \Delta_{0}, \Delta_{1} \vdash C \\
& \frac{\frac{\Gamma_{0} \vdash A \Gamma_{1} \vdash B}{\Gamma_{0}, \Gamma_{1} \vdash A \otimes B} \quad \frac{\Delta_{0}, A, B, \Delta_{1} \vdash C}{\Delta_{0}, A \otimes B, \Delta_{1} \vdash C}}{\Delta_{0}, \Gamma_{0}, \Gamma_{1}, \Delta_{1} \vdash C} \leadsto \frac{\Gamma_{0} \vdash A}{\frac{\Gamma_{1} \vdash B \quad \Delta_{0}, A, B, \Delta_{1} \vdash C}{\Delta_{0}, A \Gamma_{1}, \Delta_{1} \vdash C}} \\
& \frac{\frac{\Gamma \vdash A}{\Gamma \vdash A \oplus B} \quad \frac{\Delta_{0}, A, \Delta_{1} \vdash C \quad \Delta_{0}, B, \Delta_{1} \vdash C}{\Delta_{0}, A \oplus B, \Delta_{1} \vdash C}}{\Delta_{0}, \Gamma, \Delta_{1} \vdash C} \leadsto \frac{\Gamma \vdash A \quad \Delta_{0}, A, \Delta_{1} \vdash C}{\Delta_{0}, \Gamma, \Delta_{1} \vdash c} \\
& \Gamma \vdash B \quad \Delta_{0}, A, \Delta_{1} \vdash C \quad \Delta_{0}, B, \Delta_{1} \vdash C \\
& \frac{\Gamma \vdash A \oplus B}{\frac{\Delta_{0}, A \oplus B, \Delta_{1} \vdash C}{\Delta_{0}, \Gamma, \Delta_{1} \vdash C}} \sim \frac{\Gamma \vdash B \quad \Delta_{0}, B, \Delta_{1} \vdash C}{\Delta_{0}, \Gamma, \Delta_{1} \vdash C} \\
& \frac{\frac{\Gamma \vdash A \quad \Gamma \vdash B}{\Gamma \vdash A \wedge B} \frac{\Delta_{0}, A, \Delta_{1} \vdash C}{\Delta_{0}, A \wedge B, \Delta_{1} \vdash C}}{\Delta_{0}, \Gamma, \Delta_{1} \vdash C} \leadsto \frac{\Gamma \vdash A \quad \Delta_{0}, A, \Delta_{1} \vdash C}{\Delta_{0}, \Gamma, \Delta_{1} \vdash C} \\
& \frac{\frac{\Gamma \vdash A \quad \Gamma \vdash B}{\Gamma \vdash A \wedge B}}{\Delta_{0}, \Gamma, \Delta_{1} \vdash C} \frac{\Delta_{0}, B, \Delta_{1} \vdash C}{\Delta_{0}, A \wedge B, \Delta_{1} \vdash C} \leadsto \frac{\Gamma \vdash B \quad \Delta_{0}, B, \Delta_{1} \vdash C}{\Delta_{0}, \Gamma, \Delta_{1} \vdash C} \\
& \frac{\frac{\Gamma, A \vdash B}{\Gamma \vdash A \multimap_{\mathrm{r}} B} \frac{\Delta_{1}, A \quad \Delta_{0}, B, \Delta_{2} \vdash C}{\Delta_{0}, A \circ_{\mathrm{r}} B, \Delta_{1}, \Delta_{2} \vdash C}}{\Delta_{0}, \Gamma, \Delta_{1}, \Delta_{2} \vdash C} \leadsto \frac{\frac{\Delta_{1} \vdash A \quad \Gamma, A \vdash B}{\Gamma, \Delta_{1} \vdash B} \Delta_{0}, B, \Delta_{1} \vdash C}{\Delta_{0}, \Gamma \Delta_{1}, \Delta_{2} \vdash C}
\end{aligned}
$$

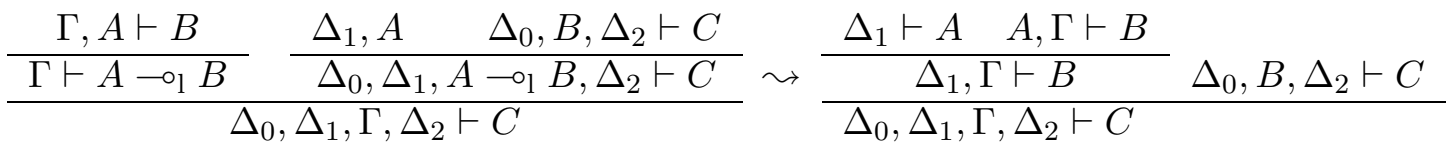


The completes the proof.

Theorem 3.2 (Cut Elimination) The Cut rule is redundant.

Proof By a simple inductive argument, it suffices to show that any proof $\pi$ of degree $d$ can be replaced by a proof of degree $<d$. We prove this by induction on the height of $\pi$.

If the last rule $r$ is not a cut rule of degree $d$ then, by the inductive hypothesis, there exist proofs $\pi_{i}$ of the premises of $r$ of degree $<d$; we can apply $r$ to these $\pi_{i}$. If the last rule is a cut rule of degree $d$ then the conditions of Lemma 3.1 apply and the result follows.

\section{The Exponential Modality}

In [GL87], Girard and Lafont introduce an exponential modality! with the following rules

$$
\begin{array}{cc}
\overline{! A \vdash \mathrm{I}}(! 1 \vdash) & \overline{! A \vdash A}(! 2 \vdash) \\
\frac{\bar{l} \boldsymbol{A \vdash ! A \otimes ! A}}{}(! 3 \vdash) & \frac{B \vdash I B \vdash A B \vdash B \otimes B}{B \vdash ! A}(\vdash !)
\end{array}
$$

The rule $(\vdash !)$ characterises the interpretation of $! A$ in any quantale as the greatest fixed point of the equation $x=I \wedge \llbracket A \rrbracket \wedge(x \otimes x)$. An important consequence of introducing! is that we regain weakening and contraction in the following form

$$
\frac{\Gamma \vdash B}{! A \vdash \mathrm{I}}(\text { Weak }) \quad \frac{\Gamma, ! A, ! A \vdash B}{\Gamma, ! A \vdash B} \text { (Con) }
$$

as derived rules of the calculus. This fact underlies the existence of the faithful translation of intuitionistic logic into intuitionistic linear logic [Gir87]. An appealing feature of NILL is that if we introduce the exponential! with the same rules $(! 1 \vdash),(! 2 \vdash),(! 3 \vdash)$ and $(\vdash !)$ then we obtain the non-commutative analogues of weakening and contraction.

Proposition 4.1 The following rules,

$$
\frac{\Gamma \vdash B}{\Gamma, ! A, \Delta \vdash B} \text { (Weak) } \frac{\Gamma, ! A, ! A, \Delta \vdash B}{\Gamma, ! A, \Delta \vdash B} \text { (Con) }
$$


are derivable in NILL.

Proof

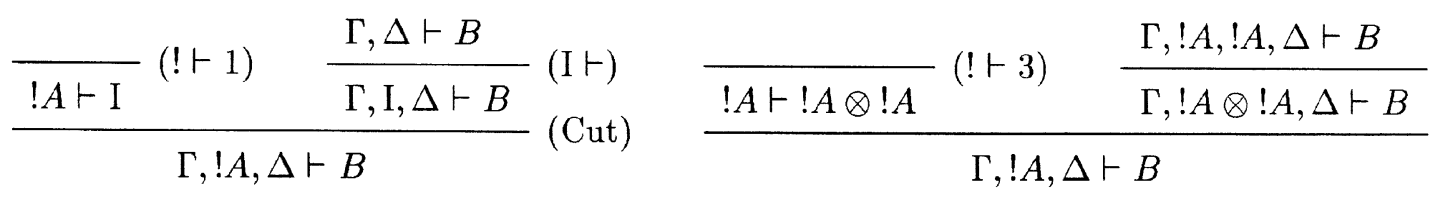

In fact, these rules are stronger than might have been expected, since a formula ! $A$ can be inserted or contracted upon at an arbitrary position in a sequent and not just at the end. Observe that the exchange rule cannot be recovered in this way since, for example, the sequent $! A, B \vdash B \otimes A$ is not derivable in NILL, as we shall demonstrate in Section 6. This accords well with the intuitive notion that the formula! $A$ represents "as many $A$ 's as one needs" [Gir89], together with the non-commutative insistence that the position in which these $A$ 's occur is significant.

\section{Quantales and NILL}

We recall some basic definitions concerning quantales [Ros90].

Definition 5.1 A quantale is a 4-tuple $\langle Q, \leq, \otimes, 1\rangle$ such that

- $\langle Q, \leq\rangle$ is a complete join semi-lattice,

- $\langle Q, \otimes, 1\rangle$ is a monoid with unit 1 , and

- for any indexing set $J$,

$$
p \otimes \vee_{j \in J} q_{j}=\vee_{j \in J}\left(p \otimes q_{j}\right) \text { and }\left(\vee_{j \in J} p_{j}\right) \otimes q=\vee_{j \in J}\left(p_{j} \otimes q\right)
$$

A quantale is commutative if $\langle Q, \otimes, 1\rangle$ is a commutative monoid.

Remark 5.2 If $\langle Q, \leq, \otimes, 1\rangle$ is a quantale then $\langle Q, \leq\rangle$ is a complete lattice, since any complete join semi-lattice is also a complete lattice, (see for example [Joh82]). 
Example 5.3 The powerset $\mathcal{P}(M)$ of a monoid $\langle M,+, 0\rangle$ is a quantale with joins given by unions, the operation $\otimes$ given by

$$
A \otimes B=\{a+b \mid a \in A, b \in B\}
$$

and unit $1=\{0\} .\langle\mathcal{P}(M), \subseteq, \otimes,\{0\}\rangle$ is the free quantale on $\langle M,+, 0\rangle$.

Definition 5.4 Let $\langle Q, \leq, \otimes, 1\rangle$ and $\left\langle Q^{\prime}, \leq^{\prime}, \otimes^{\prime}, 1^{\prime}\right\rangle$ be quantales. A function from $Q$ to $Q^{\prime}$ is a quantale homomorphism if it preserves the monoid structure and all joins, and a quantale isomorphism if it is also a bijection.

Remark 5.5 If $\langle Q, \leq, \otimes, 1\rangle$ is a quantale then the functors $q-\otimes q$ and $q \otimes-$ have right adjoints $\multimap_{\mathrm{r}}-$ and $q \multimap_{1}-$ given by

$$
q \multimap_{\mathrm{r}} p=\vee\{r \mid r \otimes q \leq p\} \text { and } q \multimap_{1} p=\vee\{r \mid q \otimes r \leq p\}
$$

In a commutative quantale these operations coincide.

The following derived operation will also prove useful.

Definition 5.6 Let $\langle Q, \leq, \otimes, 1\rangle$ be a quantale. For any $q \in Q$, let ! $q$ be the element of $Q$ given by:

$$
! q=\vee\{p \mid p \leq 1 \wedge q \wedge p \otimes p\}
$$

We now describe the interpretation of NILL in any quantale and prove soundness and completeness. Yetter [Yet90] has proved similar results for linear logic and cyclic non-commutative linear logic with respect to quantales with certain extra structure.

Definition 5.7 A model of NILL is a pair $\langle Q, \tau\rangle$ where $Q$ is a quantale and $\tau$ is a function from the linear atoms to $Q$. The interpretation of NILL in $\langle Q, \tau\rangle$ is a function $\llbracket-\rrbracket$ from the formulae $\mathcal{F}$ of NILL to $Q$ given by:

- $\llbracket A \rrbracket=\tau(A)$ for each linear atom $A$,

- $\llbracket 1 \rrbracket=\top$,

- $\llbracket \mathbf{0} \rrbracket=\perp$,

- $\llbracket \mathrm{I} \rrbracket=1$,

- $\llbracket A \wedge B \rrbracket=\llbracket A \rrbracket \wedge \llbracket B \rrbracket$, 
- $\llbracket A \oplus B \rrbracket=\llbracket A \rrbracket \vee \llbracket B \rrbracket$,

- $\llbracket A \otimes B \rrbracket=\llbracket A \rrbracket \otimes \llbracket B \rrbracket$,

- $\llbracket A \multimap_{\mathrm{r}} B \rrbracket=\llbracket A \rrbracket \multimap_{\mathrm{r}} \llbracket B \rrbracket$,

- $\llbracket A \multimap \multimap_{1} B \rrbracket=\llbracket A \rrbracket \multimap_{1} \llbracket B \rrbracket$, and

- $\llbracket ! A \rrbracket=! \llbracket A \rrbracket$

Definition 5.8 Let $\langle Q, \tau\rangle$ be a model of NILL. We say that $\langle Q, \tau\rangle$ entails the sequence $\Gamma_{1}, \Gamma_{2}, \ldots, \Gamma_{n} \vdash A$, denote $\Gamma \models_{\langle Q, \tau\rangle} A$, if $\llbracket \Gamma_{1} \rrbracket \otimes \llbracket \Gamma_{2} \rrbracket \otimes \cdots \otimes \llbracket \Gamma_{n} \rrbracket \leq$ $\llbracket A \rrbracket$. In the special case where $n=0$, we say that $\langle Q, \tau\rangle$ entails the sequent $\vdash A$ if $1 \leq \llbracket A \rrbracket$. We write $\Gamma \models A$ if $\Gamma \models_{\langle Q, \tau\rangle} A$ for all models $\langle Q, \tau\rangle$.

We now state and prove soundness and completeness of NILL with respect to interpretation in quantales. First observe that the interpretation of $\otimes$ in any model is monotonic in each argument, that is,

$$
\text { if } \llbracket A \rrbracket \leq \llbracket C \rrbracket \text { and } \llbracket B \rrbracket \leq \llbracket D \rrbracket \text { then } \llbracket A \otimes B \rrbracket \leq \llbracket C \otimes D \rrbracket \text {. }
$$

This follows immediately from fact that, in any quantale, $\otimes$ is monotonic in each argument.

Theorem 5.9 (Soundness) $\Gamma \vdash A$ implies that $\Gamma \models A$.

Proof: We proceed by induction on the structure of the proof of $\Gamma \vdash A$. Thus we show soundness with respect to each of the rules of non-commutative intuitionistic linear logic.

In the case of the rule (Id), we have $\llbracket A \rrbracket \leq \llbracket A \rrbracket$ for any formula $A$.

In the case of the rule (Cut), by inductive hypothesis $\llbracket \Gamma \rrbracket \leq \llbracket A \rrbracket$ and $\llbracket \Delta \otimes$ $A \otimes \Delta^{\prime} \rrbracket \leq \llbracket B \rrbracket$.

By monotonicity of $\otimes$, we have $\llbracket \Delta \otimes \Gamma \otimes \Delta^{\prime} \rrbracket \leq \llbracket B \rrbracket$.

In the case of the rule $(\mathbf{0} \vdash)$, we have $\llbracket \Gamma \rrbracket \otimes \perp_{Q} \otimes \llbracket \Delta \rrbracket=\perp_{Q} \leq \llbracket A \rrbracket$ for any formula $A$.

In the case of the rule $(\vdash \mathbf{1})$, the proof is immediate since $\mathbf{1}$ is interpreted by the top element of the quantale.

In the case of the rule $(\mathrm{I} \vdash)$, by hypothesis we have $\llbracket \Gamma \rrbracket \otimes \llbracket \Delta \rrbracket \leq \llbracket A \rrbracket$. 
Since 1 is the unit of $\otimes$, we have $\llbracket \Gamma \rrbracket \otimes 1 \otimes \llbracket \Delta \rrbracket \leq \llbracket A \rrbracket$ and so $\llbracket \Gamma \rrbracket \otimes \llbracket \mathrm{I} \rrbracket \otimes \llbracket \Delta \rrbracket \leq \llbracket A \rrbracket$ and so

$\llbracket \Gamma \otimes \mathrm{I} \otimes \Delta \rrbracket \leq \llbracket A \rrbracket$ as required.

In the case of the rule $(\vdash \mathrm{I})$, the result is immediate.

In the case of the rule $(\otimes \vdash)$ the result is immediate.

In the case of the rule $(\vdash \otimes)$, by inductive hypothesis $\llbracket \Gamma \rrbracket \leq \llbracket A \rrbracket$ and $\llbracket \Delta \rrbracket \leq$ $\llbracket B \rrbracket$.

By monotonicity of $\otimes$, we have $\llbracket \Gamma \otimes \Delta \rrbracket \leq \llbracket A \otimes B \rrbracket$.

In the case of the rule $(\vdash \oplus \mathrm{l})$, by inductive hypothesis $\llbracket \Gamma \rrbracket \leq \llbracket A \rrbracket$.

For any formula $B$ we have $\llbracket \Gamma \rrbracket \leq \llbracket A \rrbracket \vee \llbracket B \rrbracket$ and so $\llbracket \Gamma \rrbracket \leq \llbracket A \rrbracket \vee \llbracket B \rrbracket=\llbracket A \oplus B \rrbracket$.

In the case of the rule $(\vdash \oplus r)$, the proof is similar.

In the case of $(\oplus \vdash)$, by inductive hypothesis $\llbracket \Gamma \rrbracket \otimes \llbracket A \rrbracket \otimes \llbracket \Delta \rrbracket \leq \llbracket C \rrbracket$ and $\llbracket \Gamma \rrbracket \otimes \llbracket B \rrbracket \otimes \llbracket \Delta \rrbracket \leq \llbracket C \rrbracket$. Hence $\llbracket \Gamma \rrbracket \otimes(\llbracket A \rrbracket \wedge \llbracket B \rrbracket) \otimes \llbracket \Delta \rrbracket \leq \llbracket C \rrbracket$ and $\llbracket \Gamma \otimes(A \oplus B) \otimes \Delta \rrbracket \leq \llbracket C \rrbracket$.

In the case of the rule $(\vdash \wedge)$, by inductive hypothesis $\llbracket \Gamma \rrbracket \leq \llbracket A \rrbracket$ and $\llbracket \Gamma \rrbracket \leq \llbracket B \rrbracket$ and thus $\llbracket \Gamma \rrbracket \leq \llbracket A \rrbracket \wedge \llbracket B \rrbracket=\llbracket A \wedge B \rrbracket$.

In the case of the rule $(1 \wedge \vdash)$, by inductive hypothesis $\llbracket \Gamma \rrbracket \otimes \llbracket A \rrbracket \otimes \llbracket \Delta \rrbracket \leq \llbracket C \rrbracket$, and so we have $\llbracket \Gamma \rrbracket \otimes(\llbracket A \rrbracket \wedge \llbracket B \rrbracket) \otimes \llbracket \Delta \rrbracket \leq \llbracket \Gamma \rrbracket \otimes \llbracket A \rrbracket \otimes \llbracket \Delta \rrbracket$ for any formula $B$. Thus $\llbracket \Gamma \rrbracket \otimes(\llbracket A \wedge B \rrbracket) \otimes \llbracket \Delta \rrbracket \leq \llbracket C \rrbracket$ by definition of the interpretation of $\wedge$, and so $\llbracket \Gamma \otimes(A \wedge B) \otimes \Delta \rrbracket \leq \llbracket C \rrbracket$.

In the case of the rule $(\mathrm{r} \wedge \vdash)$ the proof is similar.

In the case of rule $\left(\multimap_{\mathrm{r}} \vdash\right)$, by inductive hypothesis $\llbracket \Gamma \rrbracket \leq \llbracket A \rrbracket$ and $\llbracket \Delta \otimes B \otimes$ $\Delta^{\prime} \rrbracket \leq \llbracket C \rrbracket$.

Now $\vee\{Y \mid Y \otimes \llbracket A \rrbracket \leq \llbracket B \rrbracket\} \leq \vee\{Z \mid Z \otimes \llbracket \Gamma \rrbracket \leq \llbracket B \rrbracket\}$, since $\llbracket \Gamma \rrbracket \leq \llbracket A \rrbracket$.

Thus $\llbracket\left(A \multimap \multimap_{\mathrm{r}} B\right) \rrbracket \otimes \llbracket \Gamma \rrbracket \leq \llbracket B \rrbracket$ and so

$\llbracket\left(A \multimap \multimap_{\mathrm{r}} B\right) \otimes \Gamma \rrbracket \leq \llbracket B \rrbracket$ and so

$\llbracket \Delta \otimes(A \multimap \mathrm{r} B) \otimes \Gamma \otimes \Delta^{\prime} \rrbracket \leq \llbracket \Delta \otimes B \otimes \Delta^{\prime} \rrbracket$ by monotonicity, and so $\llbracket \Delta \otimes\left(A \multimap \multimap_{\mathrm{r}} B\right) \otimes \Gamma \otimes \Delta^{\prime} \rrbracket \leq \llbracket C \rrbracket$.

In the case of the rule $\left(\multimap_{1} \vdash\right)$, the proof is similar. 
In the case of the rule $\left(\vdash \multimap_{\mathrm{r}}\right)$, by inductive hypothesis $\llbracket \Gamma \otimes A \rrbracket \leq \llbracket B \rrbracket$. Hence $\llbracket \Gamma \rrbracket \leq \vee\{Y \mid Y \otimes \llbracket A \rrbracket \leq \llbracket B \rrbracket\}$, and so $\llbracket \Gamma \rrbracket \leq \llbracket A \multimap \multimap_{\mathrm{r}} B \rrbracket$.

In the case of the rule $\left(\vdash \varlimsup_{1}\right)$, the proof is similar.

In the case of the rule $(! \vdash)$, observe that since

$$
\llbracket ! A \rrbracket=\vee\{B \mid B \leq 1 \wedge \llbracket A \rrbracket \wedge(B \otimes B)\},
$$

it is immediate that $\llbracket ! A \rrbracket \leq \llbracket \mathrm{I} \wedge A \wedge(! A \otimes ! A) \rrbracket$.

In the case of the rule $(\vdash$ !), by inductive hypothesis $\llbracket B \rrbracket \leq \llbracket \mathrm{I} \wedge A \wedge(B \otimes B) \rrbracket$. Hence

$$
\llbracket B \rrbracket \leq \vee\{Y \mid Y \leq 1 \wedge \llbracket A \rrbracket \wedge(Y \otimes Y)\},
$$

and so $\llbracket B \rrbracket \leq \llbracket ! A \rrbracket$.

This completes the proof that linear logic is sound with respect to interpretation in quantales.

In order to prove completeness, we construct a model $\langle Q, \tau\rangle$ for which $\Gamma \models\langle Q, \tau\rangle$ $A$ implies that $\Gamma \vdash A$. The following lemmas will prove useful.

Lemma $5.10 \Gamma_{1}, \Gamma_{2}, \ldots, \Gamma_{n} \vdash A$ if and only if $\Gamma_{1} \otimes \Gamma_{2} \otimes \ldots \otimes \Gamma_{n} \vdash A$.

Proof: Suppose that $\Gamma_{1}, \Gamma_{2}, \ldots, \Gamma_{n} \vdash A$. Then by repeated applications of the rule $(\otimes \vdash)$, we obtain $\Gamma_{1} \otimes \Gamma_{2} \otimes \ldots \otimes \Gamma_{n} \vdash A$.

Conversely, suppose that $\Gamma_{1} \otimes \Gamma_{2} \otimes \ldots \otimes \Gamma_{n} \vdash A$. Then by repeated applications of the rule $(\vdash \otimes)$, we obtain $\Gamma_{1}, \Gamma_{2}, \ldots, \Gamma_{n} \vdash \Gamma_{1} \otimes \Gamma_{2} \otimes \ldots \otimes \Gamma_{n}$, and then by (Cut) we obtain $\Gamma_{1}, \Gamma_{2}, \ldots, \Gamma_{n} \vdash A$.

Lemma 5.11 In NILL, we have the following results:

1. $P \otimes(Q \otimes R) H(P \otimes Q) \otimes R$,

2. $P \vdash Q \otimes \mathrm{I}$ if and only if $P \vdash Q$,

3. $P \vdash \mathrm{I} \otimes Q$ if and only if $P \vdash Q$,

4. $P \vdash A \wedge B$ if and only if $P \vdash A$ and $P \vdash B$,

5. $P \otimes Q \vdash R$ if and only if $P \vdash Q \multimap_{\mathrm{r}} R$,

6. $P \otimes Q \vdash R$ if and only if $Q \vdash P \multimap_{1} R$, and

\section{Proof:}


1. We prove $(P \otimes Q) \otimes R \vdash P \otimes(Q \otimes R)$ as follows:

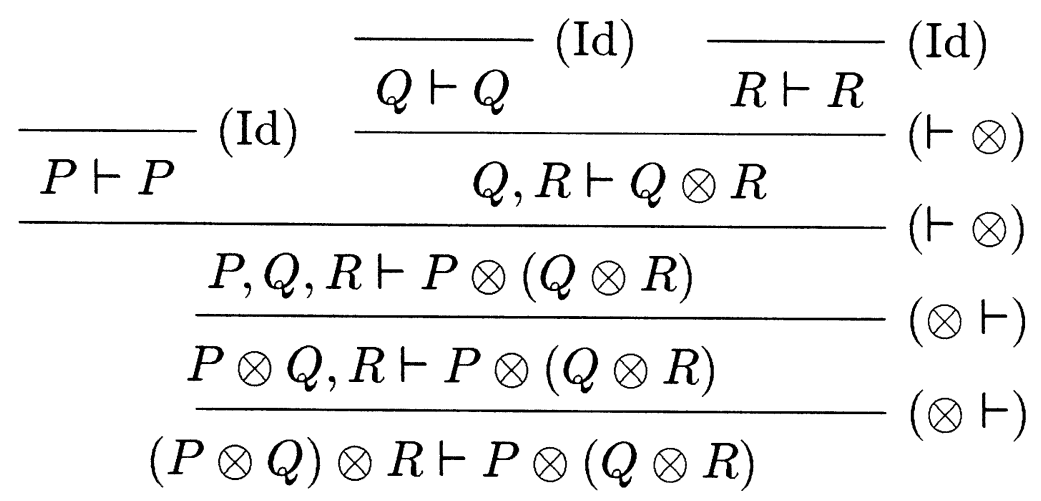

The proof of the converse is similar.

2. That $P \vdash Q \otimes \mathrm{I}$ is an immediate consequence of the rule $(\vdash \otimes)$. Conversely:

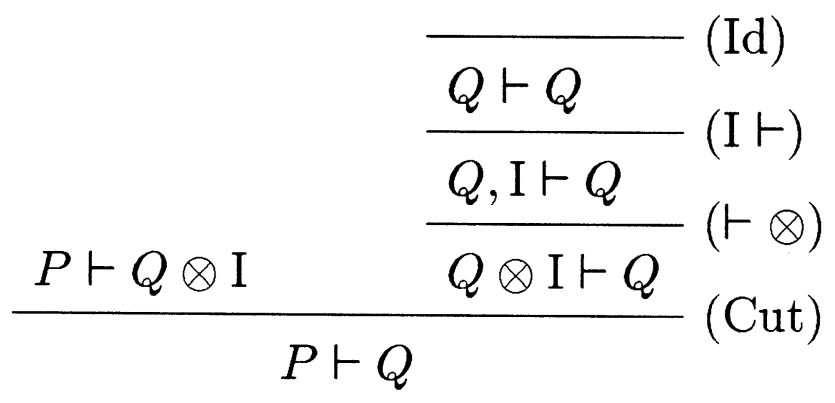

3. The proof of 3 is similar to the proof of 2 .

4. We prove $P \vdash A$ from $P \vdash A \wedge B$. The proof of $P \vdash B$ is similar. 

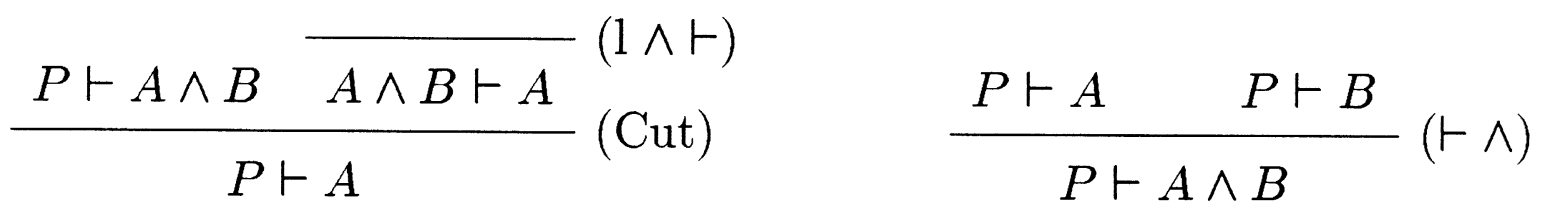

5. The rule $\left(\vdash \multimap_{r}\right)$ suffices to prove the forward implication. For the converse:

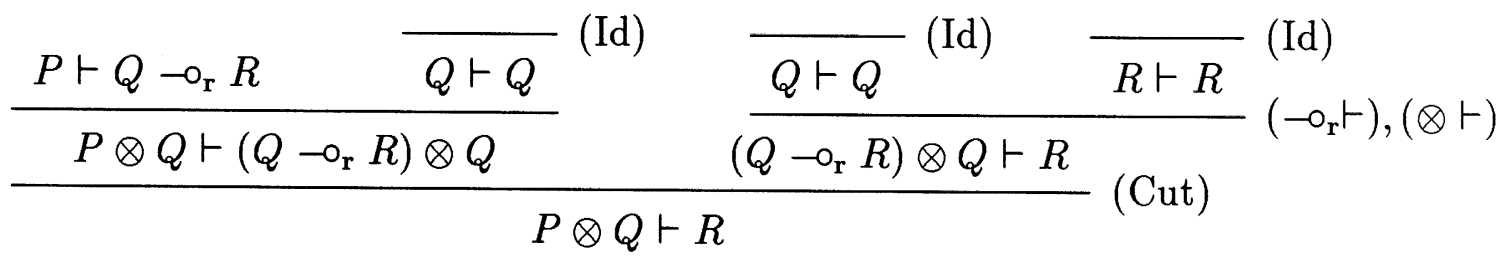

6. The proof of 6 is similar to the proof of 5 .

We now begin the construction of the required model.

Definition 5.12 Let $\mathcal{F}$ be the set of NILL formulae. A subset $\mathcal{I} \subseteq \mathcal{F}$ is an ideal if,

- $\mathbf{0} \in \mathcal{I}$

- $A \in \mathcal{I}$ and $B \vdash A$ implies that $B \in \mathcal{I}$ (that is, $\mathcal{I}$ is downwards closed), and

- $A, B \in \mathcal{I}$ implies that $A \oplus B \in \mathcal{I}$.

Notation 5.13 Let $S \subseteq \mathcal{F}$ be any subset of $\mathcal{F}$. Define

$$
\vee S=\cap\{\mathcal{I} \subseteq \mathcal{F} \mid S \subseteq \mathcal{I}\}
$$

where each $\mathcal{I}$ is an ideal. Note that $\vee S$ is an ideal since ideals are closed under intersection. 
Lemma 5.14 Let $A \in \mathcal{F}$. Then $\vee\{A\}=\{B \mid B \vdash A\}$.

Proof: Evidently $\{B \mid B \vdash A\} \subseteq \vee\{A\}$ as ideals are downwards closed. However, $\{B \mid B \vdash A\}$ is an ideal since $\mathbf{0} \vdash A$ and if $B \vdash A$ and $C \vdash A$ then $B \oplus C \vdash A$ by $(\vdash \oplus)$. Thus $\vee\{A\} \subseteq\{B \mid B \vdash A\}$.

Lemma 5.15 $\vee\{A\} \subseteq \vee\{B\}$ if and only if $A \vdash B$.

Proof: Suppose that $\vee\{A\} \subseteq \vee\{B\}$. Then by Lemma 5.14, whenever $C \vdash A$ we have $C \vdash B$. In particular, since $A \vdash A$, we have $A \vdash B$. Conversely, suppose that $A \vdash B$ and $C \in \vee\{A\}$. Then $C \vdash A$ and so, by (Cut), $C \vdash B$ and $C \in \vee\{B\}$.

Proposition 5.16 Let $Q \subseteq \mathcal{P}(\mathcal{F})$ be $\{\vee S \mid S \subseteq \mathcal{F}\}$ and let $\otimes$ be the operation given by $\vee S \otimes \vee T=\vee\{s \otimes t \mid s \in S, t \in T\}$. Then $\langle Q, \subseteq, \otimes, \vee\{I\}\rangle$ is a quantale.

Proof: The ideal $\vee\left(\bigcup_{j \in J} S_{j}\right)$ is an upper bound for $\left\{\vee S_{j} \mid j \in J\right\}$ as

$$
\vee\left(\bigcup_{j \in J} S_{j}\right)=\bigcap\left\{\mathcal{I} \subseteq \mathcal{F} \mid \bigcup_{j \in J} S_{j} \subseteq \mathcal{I}\right\} \supseteq \bigcap\left\{\mathcal{I} \subseteq \mathcal{F} \mid S_{j} \subseteq \mathcal{I}\right\} \text { for each } j
$$

Now suppose that $\vee T$ is another upper bound for $\left\{\vee S_{j} \mid j \in J\right\}$. Then $\vee S_{j} \subseteq \vee T$ for each $j$, which implies that $\bigcup_{j \in J}\left(\vee S_{j}\right) \subseteq \vee T$. However,

$$
\bigcup_{j \in J}\left(\vee S_{j}\right)=\bigcup_{j \in J}\left(\bigcap\left\{\mathcal{I} \subseteq \mathcal{F} \mid S_{j} \subseteq \mathcal{I}\right\}\right)=\bigcap\left\{\mathcal{I} \subseteq \mathcal{F} \mid \bigcup_{j \in J} S_{j} \subseteq \mathcal{I}\right\}=\vee\left(\bigcup_{j \in J} S_{j}\right)
$$

and so $\vee\left(\bigcup_{j \in J} S_{j}\right)$ is the least upper bound for $\left\{\vee S_{j} \mid j \in J\right\}$.

To see that $\langle Q, \otimes, \vee\{\mathrm{I}\}\langle$ is a monoid, observe first that, by Lemma 5.11.2, $\vee S \otimes \vee\{\mathrm{I}\}=\vee\{s \otimes \mathrm{I} \mid s \in S\}=\vee\{s \mid s \in S\}=\vee S$ and similarly $\vee\{\mathrm{I}\} \otimes \vee S=$ vee $S$. Thus $\vee\{\mathrm{I}\}$ is a unit for $\otimes$. Further, using Lemma 5.11.1,

$$
\begin{aligned}
& (\vee R \otimes \vee S) \otimes \vee T=\vee\{r \otimes s\} \otimes \vee T=\vee\{(r \otimes s) \otimes t\}= \\
& \vee\{r \otimes(s \otimes t)\}=\vee R \otimes(\vee S \otimes \vee T)
\end{aligned}
$$

and so $\otimes$ is associative and $\langle\mathcal{Q}, \otimes, \vee\{\mathrm{I}\}\rangle$ is a monoid.

Finally,

$$
\begin{aligned}
\vee S \otimes \vee_{j \in J}\left(\vee T_{j}\right) & =\vee S \otimes \operatorname{vee}\left(\cup_{j \in J} T_{j}\right) \\
& =\vee\left\{s \otimes t \mid s \in S, t \in \cup_{j \in J} T_{j}\right\}
\end{aligned}
$$




$$
\begin{aligned}
& =\vee\left(\cup_{j \in J}\left\{s \otimes t \mid s \in S, t \in T_{j}\right\}\right) \\
& =\vee_{j \in J}\left(\vee\left\{s \otimes t \mid s \in S, t \in T_{j}\right\}\right) \\
& =\vee_{j \in J}\left(\vee S \otimes \vee T_{j}\right)
\end{aligned}
$$

and similarly $\vee_{j \in J}\left(\vee T_{j}\right) \otimes \vee S=\vee_{j \in J}\left(\vee T_{j} \otimes \vee S\right)$.

Proposition 5.17 Third exists a model $\langle Q, \tau\rangle$ such that $\llbracket P \rrbracket=\vee\{P\}$ for all $P \in \mathcal{F}$.

Proof: Let $Q$ be the quantale constructed in Proposition 5.16 and, for each atom $A$, define $\tau(A)=\vee\{A\}$. We prove by induction on the structure of the formula $P$ that $\llbracket P \rrbracket=\vee\{P\}$.

- $\llbracket A \rrbracket=\vee\{A\}$ by definition, for any atom $A$.

- $\llbracket \mathbf{1} \rrbracket=\top_{Q}=\mathcal{F}=\{B \in \mathcal{F} \mid B \vdash \mathbf{1}\}=\vee\{\mathbf{1}\}$.

- $\llbracket 0 \rrbracket=\perp_{Q}=\{B \in \mathcal{F} \mid B \vdash \mathbf{0}\}=\vee\{\mathbf{0}\}$ since every ideal contains $\mathbf{0 .}$

- $\llbracket \mathrm{I} \rrbracket=1=\vee\{\mathrm{I}\}$ by definition.

$$
\begin{aligned}
\llbracket A \otimes B \rrbracket & =\llbracket A \rrbracket \otimes \llbracket B \rrbracket \\
& =\vee\{A\} \otimes \vee\{B\} \quad \text { by the inductive hypothesis } \\
& =\vee\{A \otimes B\} \text { by definition }
\end{aligned}
$$

$$
\begin{aligned}
\llbracket A \wedge B \rrbracket & =\llbracket A \rrbracket \cap \llbracket B \rrbracket \\
& =\vee\{A\} \cap \vee\{B\} \quad \text { by the inductive hypothesis } \\
& =\{C \mid C \vdash A \text { and } C \vdash B\} \text { by Lemma } 5.14 \\
& =\{C \mid C \vdash A \wedge B\} \text { by Lemma } 5.11 .4 \\
& =\vee\{A \wedge B\} \text { by Lemma } 5.14
\end{aligned}
$$

$$
\begin{aligned}
\llbracket A \oplus B \rrbracket & =\llbracket A \rrbracket \vee \llbracket B \rrbracket \\
& =\vee\{A\} \vee \vee\{B\} \quad \text { by the inductive hypothesis } \\
& =\vee\{A, B\} \\
& =\vee\{A \otimes B\} \quad \text { since }\{A, B\} \subseteq \mathcal{I} \text { iff } A \oplus B \in \mathcal{I}
\end{aligned}
$$




$$
\begin{aligned}
\llbracket A \multimap \multimap_{\mathrm{r}} B \rrbracket & =\llbracket A \rrbracket \multimap_{\mathrm{r}} \llbracket B \rrbracket \\
& =\vee\{A\} \multimap_{\mathrm{r}} \vee\{B\} \quad \text { by the inductive hypothesis } \\
& =\vee\{\vee S \mid \vee S \otimes \vee\{A\} \subseteq \vee\{B\}\} \\
& =\vee(\cup\{S \mid \vee S \otimes \vee\{A\} \subseteq \vee\{B\}\}) \text { by Proposition 5.16 } \\
& =\vee(\cup\{\{C\} \mid \vee\{C\} \otimes \vee\{A\} \subseteq \vee\{B\}\}) \\
& =\vee\{C \mid \vee\{C\} \otimes \vee\{A\} \subseteq \vee\{B\}\} \\
& =\vee\{C \mid \vee\{C \otimes A\} \subseteq \vee\{B\}\} \\
& =\vee\{C \mid C \otimes A \vdash B\} \text { by Lemma 5.15 } \\
& =\vee\{C \mid C \vdash A \multimap \mathrm{r} B\} \text { by Lemma 5.11.5 } \\
& =\vee\left(\vee\left\{A \multimap_{\mathrm{r}} B\right\}\right) \\
& =\vee\left\{A \multimap_{\mathrm{r}} B\right\} .
\end{aligned}
$$

$$
\begin{aligned}
\llbracket A \multimap 1 ~ B \rrbracket & =\llbracket A \rrbracket \multimap_{1} \llbracket B \rrbracket \\
& =\vee\{A\} \multimap_{1} \vee\{B\} \quad \text { by the inductive hypothesis } \\
& =\vee\{\vee S \mid \vee\{A\} \otimes \vee S \subseteq \vee\{B\}\} \\
& =\vee(\cup\{S \mid \vee\{A\} \otimes \vee S \subseteq \vee\{B\}\}) \text { by Proposition 5.16 } \\
& =\vee(\cup\{\{C\} \mid \vee\{A\} \otimes \vee\{C\} \subseteq \vee\{B\}\}) \\
& =\vee\{C \mid \vee\{A\} \otimes \vee\{C\} \subseteq \vee\{B\}\} \\
& =\vee\{C \mid \vee\{A \otimes C\} \subseteq \vee\{B\}\} \\
& =\vee\{C \mid A \otimes C \vdash B\} \text { by Lemma 5.15 } \\
& =\vee\{C \mid C \vdash A \multimap B\} \text { by Lemma } 5.16 \\
& =\vee\left(\vee\left\{A \multimap \multimap_{1} B\right\}\right) \\
& =\vee\left\{A \multimap \multimap_{1} B\right\} .
\end{aligned}
$$

$$
\begin{aligned}
\llbracket ! A \rrbracket & =! \llbracket A \rrbracket \\
& =!(\vee\{A\}) \quad \text { by the inductive hypothesis } \\
& =\vee\{\vee S \mid \vee S \subseteq \vee\{\mathrm{I}\} \cap \vee\{A\} \cap(\vee S \otimes \vee S)\} \\
& =\vee(\cup\{S \mid \vee S \subseteq \vee\{\mathrm{I}\} \cap \vee\{A\} \cap(\vee S \otimes \vee S)\}) \text { by Prop. } 5.16 \\
& =\vee(\cup\{\{C\} \mid \vee\{C\} \subseteq \vee\{\mathrm{I}\} \cap \vee\{A\} \cap(\vee\{C\} \otimes \vee\{C\})\}) \\
& =\vee\{C \mid \vee\{C\} \subseteq \vee\{\mathrm{I}\} \cap \vee\{A\} \cap(\vee\{C\} \otimes \vee\{C\})\} \\
& =\vee\{C \mid \vee\{C\} \subseteq \vee\{\mathrm{I}\} \cap \vee\{A\} \cap(\vee\{C \otimes C\}\} \\
& =\vee\{C \mid C \vdash \mathrm{I} \cap A \cap C \otimes C\} \text { by Lemma } 5.15 \\
& =\vee(\vee\{! A\}) \\
& =\vee\{! A\} .
\end{aligned}
$$


This completes the proof.

Theorem 5.18 (Completeness) If $\Gamma \models A$ then $\Gamma \vdash A$

Proof: Consider the quantale constructed in Proposition 5.16. By Lemma 5.10, it suffices to show that the interpretation $\llbracket-\rrbracket$ of Proposition 5.17 is such that for all $A$ and $B$ in $\mathcal{F}, \llbracket A \rrbracket \subseteq \llbracket B \rrbracket$ implies that $A \vdash B$. However, by Proposition 5.17, for each $A$ in $\mathcal{F}, \llbracket A \rrbracket=\vee\{A\}$ and therefore, by Lemma 5.15, $A \rrbracket \subseteq \llbracket B \rrbracket$ if and only if $A \vdash B$.

\section{Relational Models of NILL}

An interesting class of quantales comprises those in which the elements are relations on a set $\mathrm{A}$ ordered by inclusion, and the monoid operation is relational composition. In this section, we prove our central result, that relational quantales provide a sound and complete class of models for NILL. We obtain this result by considering quantales which consist of a subset of the relations on a set $A$.

Definition 6.1 Let $A$ be a set. A relational quantale on $A$ is a pair $\langle\mathcal{Q}, \mathrm{I}\rangle$ where $\mathcal{Q} \subset \mathcal{P}(A \times A)$ and $I \in \mathcal{Q}$ such that:

- $\langle\mathcal{Q}, \subseteq\rangle$ is a complete join semi-lattice,

- $\langle\mathcal{Q}, \circ, \mathrm{I}\rangle$ is a monoid,

- for any indexing set $J$,

$$
p \circ \vee_{j \in J} q_{j}=\vee_{j \in J}\left(p \circ q_{j}\right) \text { and }\left(\vee_{j \in J} p_{j}\right) \circ q=\vee_{j \in J}\left(p_{j} \circ q\right)
$$

where $\circ$ is relational composition, that is $R \circ S=\{\langle a, b\rangle \mid \exists c \cdot\langle a, c\rangle \in R$ and $\langle c, b\rangle \in S\}$.

Remark 6.2 Note that in a relational quantale, while we have $\cup A_{j} \subseteq \vee A_{j}$, we du not in general have equality, since $\cup A_{j}$ may not be an element of $\mathcal{Q}$.

A relational quantale $\langle\mathcal{Q}, \mathrm{I}\rangle$ is commutative if $\langle\mathcal{Q}, \circ, \mathrm{I}\rangle$ is a commutative monoid. It is immediate that any (commutative) relational quantale is a (commutative) quantale. 
Example 6.3 Let $A$ be any set and let $\Delta_{A}$ be the diagonal relation $\{\langle a, a\rangle \mid$ $a \in A\}$, Then $\left\langle\mathcal{P}(A \times A), \Delta_{A}\right\rangle$ is a relational quantale in which joins are given by unions. Hoare and He Jefing [HH87] use this example to model non-deterministic while programs.

Example 6.4 Let $\mathcal{J}$ be the closed interval $[0,1]$ of the real line, and let $\mathcal{Q}$ be the set of subsets of $\mathcal{J} \times \mathcal{J}$ of the form,

$$
\{\langle x, y\rangle \mid y \leq a \cdot x\}
$$

where $a \in[0,1]$. That is, $\mathcal{Q}$ consists of all the shaded areas of the form:

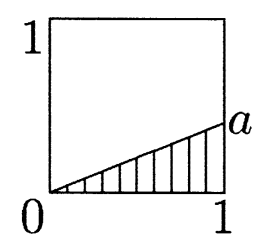

Let $I=\{\langle x, y\rangle \mid y \leq x\}$. Then $\langle\mathcal{Q}, I\rangle$ is a commutative relational quantale, in which non-empty finite joins are given by unions, and the bottom element is the set $\{\langle x, y\rangle \mid y=0\}$. Relational composition gives:
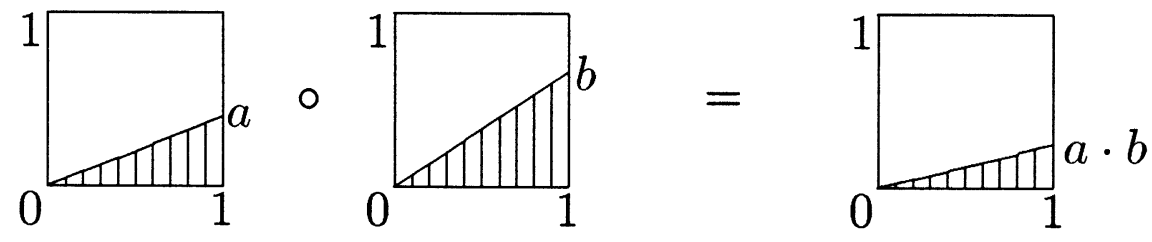

We now recall the following important result from [BG91].

Theorem 6.5 Every quantale is isomorphic to a relational quantale on its underlying set.

Proof: (sketch) Let $\langle Q, \leq, \otimes, 1\rangle$ be a quantale. We write

$$
\hat{r}=\{\langle p, q\rangle \mid p \leq r \otimes q\} \text { and } \widehat{Q}=\{\hat{r} \mid r \in Q\} .
$$

With the above notation, we show that $\hat{r} \subseteq \hat{s}$ if and only if $r \leq s$. If $r \leq s$ and $\langle p, q\rangle \in \hat{r}$ then $p \leq r \otimes q$, whence $p \leq s \otimes q$ whence $\langle p, q\rangle \in \hat{s}$ : thus 
$\hat{r} \subseteq \hat{s}$. Conversely, $\langle r, 1\rangle \in \hat{r}$ since $r \leq r \otimes 1=r$. If $\hat{r} \subseteq \hat{s}$ then $\langle r, 1\rangle \in \hat{s}$, which implies that $r \leq s \otimes 1=s$.

In addition, we show that $\langle\widehat{Q}, \subseteq\rangle$ a complete join semilattice with $\vee \widehat{r_{j}}=\widehat{\nabla r_{j}}$. By definition, $r_{j} \leq \vee r_{j}$ for each $j$, and so $\widehat{r_{j}} \subseteq \widehat{\nabla r_{j}}$ for each $j$. Therefore, $\widehat{\nabla r_{j}}$ is an upper bound for $\left\{\widehat{r}_{j}\right\}$. If $\hat{s}$ is another upper bound then $\widehat{r_{j}} \subseteq \hat{s}$ for each $j$ and $r_{j} \leq s$ for each $j$ whence $\vee r_{j} \leq s$ and so $\widehat{\nabla r_{j}} \subseteq \hat{s}$. Thus $\widehat{\nabla r_{j}}$ is the least uiper bound of $\left\{\widehat{r}_{j}\right\}$.

Further, observe that $\exists q .\left(p \leq r \otimes q\right.$ and $\left.q \leq s \otimes p^{\prime}\right)$ if and only if $p \leq$ $(r \otimes s) \otimes p^{\prime}$, since if there exists $q$ such that $p \leq r \otimes q$ and $q \leq s \otimes p^{\prime}$, then $p \leq r \otimes q \leq r \otimes\left(s \otimes p^{\prime}\right)=(r \otimes s) \otimes p^{\prime}$.

Conversely, if $p \leq(r \otimes s) \otimes p^{\prime}$ then, taking $q=s \otimes p^{\prime}, q \leq s \otimes p^{\prime}$ and $p \leq(r \otimes s) \otimes p^{\prime}=r \otimes\left(s \otimes p^{\prime}\right)=r \otimes q$.

Therefore, $\hat{r} \circ \hat{s}=\{\langle p, q\rangle \mid p \leq r \otimes q\} \circ\{\langle p, q\rangle \mid p \leq s \otimes q\}$

$$
\begin{aligned}
& =\left\{\left\langle p, p^{\prime}\right\rangle \mid \exists q .\left(p \leq r \otimes q \text { and } q \leq s \otimes p^{\prime}\right)\right\} \\
& =\left\{\left\langle p, p^{\prime}\right\rangle \mid p \leq(r \otimes s) \otimes p^{\prime}\right\}=\widehat{r \otimes s} .
\end{aligned}
$$

Now $\langle\widehat{Q}, \hat{1}\rangle$ is a relational quantale on $Q$ as $\langle\widehat{Q}, \subseteq\rangle$ is a complete join semilattice, $\widehat{Q}$ is closed under $\circ$ and $\hat{1}$ is the unit of $\circ$ ( since $\hat{r} \circ \hat{1}=\widehat{r \otimes 1}=\hat{r}$ and $\hat{q} \circ \hat{r}=\widehat{1 \otimes r}=\hat{r}$ ) and $\circ$ distributes over joins on both sides, since

$$
\left.\hat{s} \circ \vee \widehat{r}_{j}=\hat{s} \circ \widehat{\nabla r}_{j}=\widehat{s \otimes \vee r_{j}}=\vee{\widehat{\left(s \otimes r_{j}\right.}}\right)=\vee\left(\widehat{s \otimes r_{j}}\right)=\vee\left(\hat{s} \circ \widehat{r}_{j}\right)
$$

and similarly, $\left(\vee \widehat{r_{j}}\right) \circ \hat{s}=\vee\left(\widehat{r_{j}} \circ \hat{s}\right)$.

Finally, the function $(\widehat{-})$ from $Q$ to $\widehat{Q}$ mapping $r$ to $\hat{r}$ is an isomorphism of quantales.

Corollary 6.6 Every commutative quantale is isomorphic to a commutative relational quantale on its underlying set.

Proof: Follows from the proof of Theorem 6.5 and the observation that if $\langle Q, \leq, \otimes 1\rangle$ is a commutative quantale, then $\hat{r} \circ \hat{s}=\hat{s} \circ \hat{r}$, since $\hat{r} \circ \hat{s}=\widehat{r \otimes s}=$ $\widehat{s \otimes r}=\hat{s} \circ \hat{r}$.

We now prove the completeness of NILL with respect to interpretation in relational quantales. Soundness follows from Theorem 5.9 as every relational quantale is a quantale. 
Notation 6.7 We say that a model $\langle\widehat{Q}, \hat{\tau}\rangle$ is relational if $Q$ is a relational quantale. We write $\Gamma \models^{R} A$ if $\Gamma \models{ }_{\langle\hat{Q}, \hat{\tau}\rangle} A$ for all relational models $\langle Q, \tau\rangle$.

Proposition 6.8 $\Gamma \models A$ if and only if $\Gamma \models{ }^{R} A$.

Proof: Let $Q$ be a quantale and $\tau$ an interpretation of the linear atoms in $Q$. Let $\hat{\tau}$ be the function from linear atoms to $\widehat{Q}$ given by $\hat{\tau}(A)=\widehat{\tau(A)}$. It follows immediately from Theorem 6.5 that $\Gamma \models_{\langle Q, \tau\rangle} A$ if and only if $\Gamma \models_{\langle Q, \tau\rangle} A$.

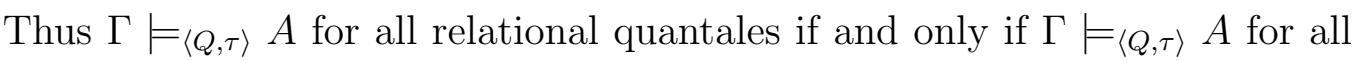
quantales.

Corollary 6.9 (Completeness for relational quantales) If $\Gamma \models^{R} A$ then $\Gamma \vdash A$.

Proof: Follows immediately from Theorem 5.18 and Proposition 6.8

Remark 6.10 A similar argument implies that intuitionistic linear logic is complete with respect to interpretation in commutative relatiunal quantales.

As an example, we exhibit a relational quantale with elements $a$ and $b$ in which $! a \circ b \nsubseteq b \circ a$. It follows, as we mentioned in Section 4 , that the sequent $! A, B \vdash B \otimes A$ is not derivable in NILL.

Example 6.11 Let $A$ be a 2-element set. Let $\mathrm{Q}$ be the set of relations represented below:
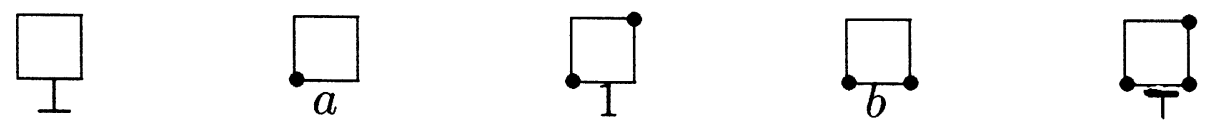

It is readily verified that $Q$ is a relational quantale with lattice structure given by:

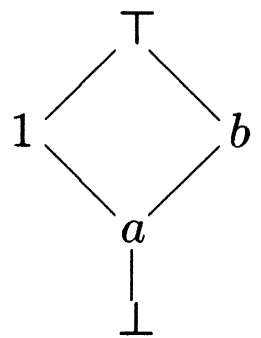

and in which relational composition gives, 


\begin{tabular}{c|ccccc}
$\circ$ & $\perp$ & $a$ & 1 & $b$ & $\top$ \\
\hline$\perp$ & $\perp$ & $\perp$ & $\perp$ & $\perp$ & $\perp$ \\
$a$ & $\perp$ & $a$ & $a$ & $b$ & $b$ \\
1 & $\perp$ & $a$ & 1 & $b$ & $\top$ \\
$b$ & $\perp$ & $a$ & $b$ & $b$ & $b$ \\
$\top$ & $\perp$ & $a$ & $\top$ & $b$ & $\top$.
\end{tabular}

In this quantale we have $! a=a$ and $! a \circ b=a \circ b=b$, while $b \circ a=a$. Thus in this case, the relation $! a \circ b$ is strictly greater than $b \circ a$.

\section{Classical Linear Logic}

We have described a system of non-commutative intuitionistic linear logic and proved its completeness with respect to interpretation in relational quantales.

A natural extension of this work is to investigate non-commutative classical linear logic. In classical linear logic one has an involutive negation $\neg$ and an additional connective "par" ( $\square$ ) satisfying the de Morgan duality $A \square B=$ $\neg(\neg A \otimes \neg B)$.

In relational quantales a natural candidate for $\square$ is the operation defined by $R \square S=\{\langle a, b\rangle \mid \forall c .\langle a, c\rangle \in R$ or $\langle c, b\rangle \in S\}$. If a relational quantale $\mathcal{Q}$ is closed under the operation of complementation and we define $\neg R$ to be the complement of $R$ then $\mathcal{Q}$ is closed under $\square$ and we have $R \square S=\neg(\neg R \circ \neg S)$. Thus one could model classical linear logic in any relational quantale which is closed under complementation. However, not all relational quantales are closed under complementation, and further-more closure under $\square$ does not entail closure under complementation. For example, the relational quantale of Example 6.4 is closed under $\square$, as it satisfies $R \square S=R \circ S$, but is certainly not closed under complementation. Indeed, the only involutive operation $\neg$ on this relational quantale satisfying $R \square S=\neg(\neg R \circ \neg S)$ is the identity.

At present, we lack a representation theorem for the quantales with the additional structure required to interpret classical linear logic. However, we are hopeful that this problem can be solved and will establish a noncommutative classical linear logic. 


\section{Acknowledgements}

This work has benefited considerably from the comments and suggestions of Simon Ambler, David Pym and Glynn Winskel.

\section{References}

[BG91] C. T. Brown and D. J. Gurr, A Representation Theorem for Quantales, to appear in Jour. of Pure und Applied Algebra.

[Gir87] J-Y. Girard, Linear Logic, TCS, 50:1-102, 1987.

[Gir89] J-Y. Girard, Towards a Geometry of Interaction, in Categories in Computer Science end Logic, AMS volume 92, 1989.

[GL87] J-Y. Girard and Y. Lafont, Linear Logic end Lazy Computation, in Proc. TAPSOFT 87 (Pisa), volume 2, pages 52-66, 1987, SpringerVerlag,(LNCS 250).

[GLT89] J.-Y. Girard, Y. Lafont and P. Taylor, Proofs and Types, CUP, 1989, Cambridge Tracts in Theoretical Computer Science 7.

[HH87] C. A. R. Hoare and He Jefing, The Weakest Pre-Specification, in Information Processing Letters, 24:127-132, 1987.

[Joh82] P. T. Johnstone, Stone Spaces, CUP, 1982.

[Mul86] C. J. Mulvey, "\&", in Suppl. ai Rend. del Circ. Mat. di Palermo, Serie II, pp 99-104, 1986.

[Ros90] K. Rosenthal, Quantales and their Applications, Longman, 1990, Pitman Research Notes in Math. 234.

[Yet90] D. Yetter, Quantales and non-commutative Linear Logic, in Jour. Symb. Logic, 55-I:41-64, 1990. 